\title{
TAMIS sin cierre de la brecha quirúrgica
}

\author{
Diego Naiderman \\ Centro de Estudio Digestivos. Mar del Plata, Buenos Aires, Argentina. \\ Clínica Pueyrredón. Mar del Plata, Buenos Aires, Argentina. \\ H.I.G.A "Oscar Alende". Mar del Plata, Buenos Aires, Argentina.
}

\begin{abstract}
RESUMEN
Antecedentes: La cirugía mínimamente invasiva transanal (TAMIS) es una técnica que resulta especialmente útil en lesiones de recto extraperitoneal difíciles para el abordaje por vía anterior.

Actualmente sigue siendo controversial el cierre del defecto rectal por debajo de la reflexión peritoneal. La sutura endoluminal es uno de los aspectos más desafiantes del procedimiento y la literatura es discordante con respecto al cierre de la brecha quirúrgica. Nuestro objetivo es realizar un estudio descriptivo de una serie consecutiva de pacientes con lesiones de recto extraperitoneal sometidos a cirugía de TAMIS, dejando el lecho quirúrgico abierto.

Diseño: Descriptivo prospectivo longitudinal.

Material y método: Entre agosto de 2013 y julio de 2019 se incluyeron, en forma consecutiva, todos los pacientes con lesiones de recto extraperitoneal, operados con técnica de TAMIS, que presentaron lesiones benignas $\geq$ a $3 \mathrm{~cm}$ de difícil resolución endoscópica, tumores neuroendocrinos $\geq$ de $2 \mathrm{~cm}$, adenocarcinomas T1N0 sin signos histológicos de mal pronóstico, adenocarcinomas T2NO con elevado riesgo quirúrgico o que rehusaron la cirugía radical y pacientes con dudas de la remisión completa luego de la neoadyuvancia.

Todos los procedimientos fueron realizados por el mismo cirujano y en todos se dejó el lecho quirúrgico abierto.

Resultados: En un período de 6 años se trataron 35 pacientes con técnica de TAMIS, con una edad promedio de 61 años \pm 12 . El tamaño promedio de las lesiones fue de $3,68 \pm 2,1 \mathrm{~cm}(0,7-9 \mathrm{~cm})$ y la distancia desde el margen anal al extremo distal de la lesión de $5,7 \pm 1,48 \mathrm{~cm}(3$ a $8,5 \mathrm{~cm})$. El tiempo operatorio fue de $39,2 \pm 20,5 \mathrm{~min}$ y el tiempo de internación de $33 \pm 28,2$ horas. La anatomía patológica de las lesiones resecadas fue: 15 adenomas, 3 tumores carcinoides y 17 adenocarcinomas. Un ypT0, 4 carcinomas in situ, 4 tumores T1 y 8 tumores T2. En todos los casos se dejó el defecto rectal abierto, con un seguimiento mínimo postoperatorio de 9 meses.

No hubo mortalidad y la morbilidad global fue de 14,2 \% (5 pacientes). Dos pacientes fueron reinternados para el tratamiento del dolor (Clavien-Dindo II) y 3 pacientes $(8,5 \%)$ presentaron sangrado post operatorio, de los cuales solo 1 (2,8 \%) requirió reintervención (Clavien-Dindo III). No hubo complicaciones infecciosas, estenosis de recto postquirúrgicas ni perforaciones hacia la cavidad abdominal.

Conclusión: Nuestros resultados permiten describir a la técnica de TAMIS dejando la brecha quirúrgica abierta, como una técnica con buenos resultados, alta factibilidad y baja tasa de complicaciones.
\end{abstract}

Palabras claves: TAMIS; Cirugía transanal; Cierre; Brecha quirúrgica; Complicaciones

\section{ABSTRACT}

Background: Transanal Minimally Invasive Surgery (TAMIS) is a technique especially useful for extraperitoneal rectal lesions difficult to approach via the anterior approach.

Endoluminal suturing is one of the most challenging aspects of the procedure and currently the closure of the rectal defect below peritoneal reflection remains controversial and the literature is discordant regarding it.

Objective: To describe our experience with the TAMIS procedure without closure of the surgical defect in a consecutive series of patients with extraperitoneal rectal lesions.

Design: Descriptive, prospective, longitudinal study.

Material and methods: Between August 2013 and July 2019, all patients with extraperitoneal rectal lesions operated on with the TAMIS technique without closure of the surgical defect for benign lesions $\geq 3 \mathrm{~cm}$ difficult for endoscopic treatment, neuroendocrine tumors $\geq 2 \mathrm{~cm}, T 1 \mathrm{NO}$ adenocarcinomas without histological signs of poor prognosis, T2NO adenocarcinomas with high surgical risk, doubts about complete remission after neoadjuvant treatment, or patients who refused radical surgery were consecutively included. All procedures were performed by the same surgeon.

Results: In a period of 6 years, 35 patients, average age $61 \pm 12$ years, underwent the procedure. Mean size of lesions was $3.7 \pm 2.1(0.7-9) \mathrm{cm}$ and distance from the anal margin to the distal end of the lesion was $5.7 \pm 1.5(3$ to 8.5$) \mathrm{cm}$. The operative time was $39.2 \pm 20.5 \mathrm{~min}$ and the hospital stay $33 \pm 28.2 \mathrm{hs}$. There were no perforations into the abdominal cavity. Histopathology of the excised lesions was: 15 adenomas, 3 carcinoid tumors and 17 adenocarcinomas (1 ypTO, 4 carcinomas in situ, 4 T1 and 8 T2 tumors). The minimum postoperative follow-up was 9 months. There was no mortality and the overall morbidity was $14.3 \%$ (5 patients). Two patients were readmitted for pain treatment (Clavien-Dindo II) and 3 (8.5\%) had postoperative bleeding, only 1 (2.8\%) requiring reoperation (Clavien-Dindo III). There were no postoperative infectious complications or rectal stenosis.

Conclusion: This study allow us to consider the TAMIS technique without closure of the surgical defect as a procedure with good results, high feasibility and low complication rate.

Keywords: TAMIS; Transanal surgery; Closure; Surgical defect; Complications

\section{Diego Naiderman}

naidermand@hotmail.com

Recibido: mayo de 2020. Aceptado: junio de 2020

Los autores declaran no tener ningún conflicto de interés. 


\section{INTRODUCCIÓN}

La resección de recto por vía abdominal combinada con la escisión total de mesorrecto sigue siendo el tratamiento estándar para el cáncer de recto. ${ }^{1,2}$ Sin embargo, la morbilidad postoperatoria y las secuelas funcionales son altas, requiriendo una ostomía definitiva entre el 10 y el $30 \%$ de los pacientes. ${ }^{3-5}$

Los tumores benignos y malignos en estadios iniciales localizados en el recto inferior, han sido manejados tradicionalmente con escisión local, con técnica de Parks. Pero este abordaje tiene limitaciones importantes en cuanto a la exposición y visibilidad de la luz rectal. ${ }^{6}$ Buess et al. describieron en 1984 la Cirugía Endoscópica Transanal (TEM) ${ }^{7}$ y se estableció como el tratamiento de elección para los tumores de recto benignos y malignos en estadios tempranos, que no eran aptos para ser resecados con técnica de Parks o endoscopía flexible. ${ }^{8,9}$ Sin embargo, hay varios factores que impidieron que esta técnica se popularizara, como la necesidad de instrumental especial de alto costo y una larga curva de aprendizaje. .0,11 $^{10}$

La cirugía mínimamente invasiva transanal (TAMIS) descripta por Atallah et al., en 2009, ${ }^{12}$ superó estas limitaciones, utilizando un dispositivo transanal flexible e instrumental laparoscópico habitual, obteniendo resultados similares al TEM. ${ }^{13,14}$ Esta técnica resulta especialmente útil en las lesiones de recto extraperitoneal difíciles para el abordaje por vía anterior y en las que existe un bajo riesgo de perforación a la cavidad peritoneal.

Actualmente sigue siendo controversial el cierre del defecto rectal por debajo de la reflexión peritoneal, no habiendo consenso entre los cirujanos colorrectales en este punto. ${ }^{15} \mathrm{La}$ sutura endoluminal es uno de los aspectos más desafiantes del procedimiento que puede llevar más tiempo que la disección en sí misma y la inhabilidad para suturar a través de la plataforma ha sido una barrera para la adopción de esta técnica. ${ }^{16-18}$ La literatura es discordante con respecto al cierre de la brecha quirúrgica y es difícil llegar a conclusiones debido a la heterogeneidad de los trabajos, que comparan diferentes técnicas quirúrgicas, pacientes operados por cirujanos con distintos niveles de experiencia, criterios de selección dispares y decisiones dejadas a elección del cirujano interviniente. ${ }^{15,19}$

\section{OBJETIVO}

Nuestro objetivo consistió en realizar un estudio descriptivo de una serie consecutiva de pacientes con lesiones de recto extraperitoneal sometidos a cirugía de TAMIS, dejando el lecho quirúrgico abierto.

\section{DISEÑO}

Estudio descriptivo prospectivo longitudinal.

\section{MATERIALY MÉTODOS}

Entre agosto de 2013 y julio de 2019 se incluyeron, en forma consecutiva, todos los pacientes con lesiones de recto extraperitoneal, operados con técnica de TAMIS, en dos instituciones privadas y una pública de la ciudad de Mar del Plata, que presentaron lesiones benignas $\geq$ a $3 \mathrm{~cm}$ de difícil resolución endoscópica, tumores neuroendocrinos $\geq$ de $2 \mathrm{~cm}$, adenocarcinomas T1N0 sin signos histológicos de mal pronóstico, adenocarcinomas T2N0 con elevado riesgo quirúrgico o que rehusaron la cirugía radical y pacientes con dudas de la remisión completa luego de la neoadyuvancia.

La estadificación preoperatoria se realizó con tacto rectal, videocolonoscopía, resonancia nuclear magnética (RNM) de abdomen y pelvis de alta resolución de al menos 1,5 Tesla, tomografía computada de tórax (TAC) y antígeno carcinoembrionario (CEA) en las lesiones malignas. En los casos en que se realizó neoadyuvancia la reestadificación se realizó entre las 6 y 8 semanas de finalizado el tratamiento.

Todos los procedimientos fueron realizados por el mismo cirujano. Se utilizó preparación colónica con fosfatos y anestesia general y/o raquídea. La profilaxis antibiótica se realizó con metronidazol y gentamicina durante la internación, completándose en la mayoría de los casos con amoxicilina-ácido clavulánico vía oral por 5 días una vez externados.

Se usaron dispositivos transanales Sils Port $^{\circledR}$ (Covidien, Mansfield, MA, Estados unidos) o GelPointPath ${ }^{\circledR}$ (Applied Medical Rancho Santa Margarita, CA, Estados Unidos), presión de insuflación de $\mathrm{CO} 2$ de $15 \mathrm{mmHg}$, instrumental laparoscópico habitual y bisturí armónico en todos los casos (ultracision harmonic scalpel, Ethicon Endosurgery Inc. Cinncinati, OH, USA).

Se dejó el lecho quirúrgico abierto en todos los pacientes, independientemente del tamaño de la lesión o la ubicación de la misma.

El parámetro más importante a evaluar fue el de las complicaciones postoperatorias. Sangrado rectal, complicaciones infecciosas (definidas como por lo menos dos de los siguientes parámetros: fiebre mayor de 38,5 grados, dolor rectal, leucocitosis mayor de $11 \times 10^{9} \mathrm{c} / \mathrm{L}$, imágenes compatibles con colección perirrectal o diagnóstico clínico de infección), perforación hacia la cavidad peritoneal, estenosis postoperatorias que requieran algún tipo de dilatación y mortalidad.

\section{RESULTADOS}

En un período de 6 años se trataron 35 pacientes con técnica de TAMIS. Dieciocho $(51,4 \%)$ de sexo femenino, 
con una edad promedio de 61 años $\pm 12(30-86)$. El tamaño promedio de las lesiones fue de 3,68 $\pm 2,1 \mathrm{~cm}$ (rango: 0,7-9 cm) y la distancia desde el margen anal al extremo distal de la lesión de 5,7 $\pm 1,48 \mathrm{~cm}$ (rango: 3 a $8,5 \mathrm{~cm}$ ). El tiempo operatorio fue de $39,2 \pm 20,5 \mathrm{~min}$ (rango: 17-90) y el tiempo de internación de $33 \pm 28,2$ horas (rango: 1 a 7 días) (Tabla 1).

La anatomía patológica de las lesiones resecadas fue la siguiente: 15 adenomas (43\%), 3 tumores carcinoides (8 \%) y 17 adenocarcinomas (49\%). Dentro de este último grupo, hubo un paciente con ypT0, quien recibió QTyRT preoperatoria y se resecó la cicatriz para confirmar la remisión completa, 4 pacientes carcinomas in situ, 4 con tumores T1 y 8 con tumores T2 (Tabla 1). De Los pacientes con tumores T2, uno tenía biopsias de adenoma, 3 individuos se rehusaron a la cirugía radical y 4 fueron subestadificados por la resonancia, completándose en dos de ellos con operación de Miles. Cinco pacientes de la serie recibieron neoadyuvancia. En todos los casos se dejó el defecto rectal abierto, con un seguimiento mínimo postoperatorio de 9 meses.

No hubo mortalidad en la serie y la morbilidad global fue de $14,2 \%$ (5 pacientes) (Tabla 2). Dos pacientes fueron reinternados para el tratamiento del dolor, ambos casos habían recibido neoadyuvancia (Clavien-Dindo II) y 3 pacientes $(8,5 \%)$ presentaron sangrado postoperatorio: 1 paciente tuvo sangrado autolimitado al octavo día de la cirugía (Clavien-Dindo I), 1 paciente requirió transfusión con 2 unidades de glóbulos rojos (Clavien-Dindo II) y solo 1 requirió reintervención (Clavien-Dindo III) representando el 2,8 \% de los casos (Tabla 3). No hubo en la serie complicaciones infecciosas, estenosis de recto postquirúrgicas ni perforaciones hacia la cavidad abdominal.

\section{DISCUSIÓN}

El TEM fue el procedimiento que cambió radicalmente la manera de realizar la escisión transanal de lesiones rectales, ${ }^{20}$ sin embargo, varios factores impidieron que esta técnica se popularizara. ${ }^{10,11}$ La cirugía de TAMIS logró superar estas limitaciones, logrando resultados similares al TEM o incluso mejores en algunos aspectos, como el tiempo quirúrgico y la disfunción del esfínter anal. ${ }^{13,21,22}$ El paciente siempre se coloca en posición de litotomía independientemente de la localización de la lesión, permitiendo un rápido abordaje abdominal si fuera necesario. Estas ventajas, junto al menor costo y una curva de aprendizaje más corta, dieron como resultado la posibilidad de que más cirujanos incorporen las técnicas miniinvasivas de resección transanal. ${ }^{23,24}$

En nuestra serie todas las resecciones se realizaron con escisión del espesor total de la pared rectal. Esto es manda- torio para intervenciones realizadas sobre lesiones malignas y altamente recomendable en lesiones benignas, por la posibilidad de que la pieza contenga un componente invasivo, lo cual puede ocurrir hasta en un $30 \%$ de los ca$\operatorname{sos}^{25,26}$

En cuanto a la estadificación, tuvimos 4 pacientes subestadificados por RNM, de los cuales 3 habían recibido neoadyuvancia, lo que traduce la dificultad para la evaluación de estas lesiones. Si bien la ecografía transanal ha demostrado su valor en la diferenciación entre tumores T1 y T2, no fue utilizada en nuestra serie por no disponer de este método diagnóstico en nuestro medio. Por otro lado, utilizamos en todos los casos RNM de alta resolución con protocolos específicos para recto, que en los últimos años ha logrado igualar al ultrasonido transanal en la estadificación de tumores tempranos, con la ventaja de poder evaluar los factores de mal pronóstico para la resección local, así como reestadificar las lesiones luego de neoadyuvancia. ${ }^{27-30}$

A pesar de los esfuerzos por estandarizar la técnica en las resecciones transanales miniinvasivas, ${ }^{31,32}$ el cierre del de-

\section{TABLA 1: CARACTERÍSTICAS DE LA POBLACIÓN}

\begin{tabular}{lc} 
Pacientes $(\mathrm{n})$ & 35 \\
\hline Sexo femenino $(\mathrm{n}(\%))$ & $18(51,4)$ \\
\hline Edad (años) & $61 \pm 12$ \\
\hline Tamaño de la lesión $(\mathrm{cm})$ & $3,68 \pm 2,1$ \\
\hline Distancia al márgen anal $(\mathrm{cm})$ & $5,7 \pm 1,48$ \\
\hline Tiempo operatorio (min) & $39,2 \pm 20,5$ \\
\hline Tiempo de internación (hs.) & $33 \pm 28,2$ \\
\hline Anatomía patológica & \\
\hline Adenomas & 15 \\
\hline Tumor carcinoide & 3 \\
\hline Carcinoma T0 & 1 \\
\hline Carcinoma Tis. & 4 \\
\hline Carcinoma T1 & 4 \\
\hline Carcinoma T2 & 8
\end{tabular}

TABLA 2: COMPLICACIONES

Total (n (\%)) $5(14,2)$

Dolor POP (N (\%)

Sangrado $(\mathrm{n}(\%))$

Re-intervenciones (n (\%))

$1(2,8)$

TABLA 3: COMPLICACIONES: CLASIFICACIÓN DE CLAVIENDINDO

\begin{tabular}{|llll|} 
& Grado I & Grado II & Grado III \\
\hline Porcentaje & 2,8 & 8,5 & 2,8 \\
\hline Dolor POP $(n)$ & 0 & 2 & 0 \\
\hline Sangrado $(n)$ & 1 & 1 & 1
\end{tabular}


fecto rectal sigue siendo uno de los puntos más controversiales, ${ }^{20}$ especialmente por debajo de la reflexión peritoneal, donde estas técnicas son más útiles y existe menos riesgo de perforación dentro de la cavidad peritoneal. ${ }^{15}$ Los beneficios del cierre del defecto rectal no están bien dilucidados, el recto y el mesorrecto son tejidos bien vascularizados que proveen un medio excelente para el recrecimiento de la pared rectal, así como una barrera a la infección. ${ }^{33}$ Suturar el defecto es técnicamente dificultoso, ya que el espacio es muy reducido, es difícil lograr afrontar los bordes a suturar sin tensión y consume tiempo, pudiendo ser mayor que la disección en sí misma, llegando a duplicar el tiempo de la intervención. ${ }^{18-20}$ Se utilizaron distintos tipos de técnicas de sutura como intracorpóreas continuas, puntos separados con nudos extracorpóreos, clips metálicos para evitar anudar, ${ }^{16,18,33-35}$ pero aun así se considera que el $30 \%$ de los defectos no pueden cerrarse. ${ }^{20}$

Los reportes en la literatura son contradictorios, tanto en trabajos comparativos, trabajos randomizados y metaanálisis, donde en la mayoría de los mismos no se observan diferencias estadísticamente significativas en las complicaciones de quienes dejan los defectos abiertos y quienes los cierran. Hanloser et al., no encontraron diferencias significativas en las complicaciones postoperatoria (sangrado o infección), a la inversa Brown et al. encontró que los pacientes en los que se cerró el defecto después de TEM tenían menos complicaciones y menos readmisiones, aunque el grupo abierto tenía lesiones significativamente más bajas. Un tercer estudio observacional de Noura et al., se asoció con una mayor morbilidad y complicaciones más severas en el grupo en los que se cerraba el defecto. Menahem et al. en su metaanálisis sobre 503 pacientes no encontraron diferencias significativas en cuanto a la morbilidad global incluyendo sangrado e infecciones, misma conclusión a la que arribaron Lee et al., en una comunicación posterior ${ }^{15,19}$ (Tabla 4).

Existen limitaciones importantes en los trabajos publicados. La mayoría se trata de trabajos multicéntricos en los cuales no está estandarizado el manejo perioperatorio. Hay diferentes criterios de inclusión basados en altura o tamaño de las lesiones o si recibieron o no neoadyuvancia. Se comparan pacientes operados con distintas técnicas, se mencionan niveles de experiencia dispares entre los cirujanos participantes y se utilizó distinto instrumental y equipamiento. Por último, en muchos de los reportes se dejó a criterio del cirujano interviniente la decisión de cerrar o no el defecto de la pared rectal..$^{15,19,20}$

En nuestra serie todos los pacientes recibieron el mismo tratamiento perioperatorio y todos fueron operados por el mismo cirujano. Se incluyeron pacientes con y sin neoadyuvancia y en ningún caso se cerró el defecto independientemente de la altura o tamaño de la lesión. Se utilizó el bisturí armónico en todas las intervenciones, ya que se ha asociado con menor sangrado postoperatorio que solo con diatermia. ${ }^{36}$

La morbilidad global fue de 14,2 \% (5 pacientes) en línea con las series publicadas, siendo la mayoría de las complicaciones leves, presentando solo 1 (2,8 \%) grado III de la clasificación de Clavien-Dindo. Esta última, fue el único caso de la serie que requirió ser reintervenido (por TAMIS), lográndose realizar hemostasia con bisturí armónico, luego de un sangrado postoperatorio intrainternación. $\mathrm{Al}$ analizar los pacientes que recibieron neoadyuvancia en la serie, observamos que 2 de los 5 fueron readmitidos para manejo del dolor (Clavien-Dindo II). Si bien algunas series describen mayor índice de complicaciones en los pacientes que recibieron quimio-radioterapia, la resección local tiene una indicación precisa y es muy útil en los casos de duda de remisión completa, siendo segura desde el punto de vista oncológico. ${ }^{37-39}$ Nuestra serie permitió identificar tumor residual en 2 pacientes a quienes se les realizó posteriormente amputación abdominoperineal. Se describió una tasa de dehiscencia de sutura en los pacientes en que se cierra el defecto del $47 \%$ y específicamente en los pacientes con neoadyuvancia cercana al $60 \%$, pero no está claro si esto tiene alguna relación con el dolor postoperatorio, existiendo incluso la posibilidad de que dejar abierta la brecha para el cierre por segunda intención podría disminuir esta complicación. ${ }^{37}$

No tuvimos infecciones postoperatorias, lo cual podría asociarse a que, al no cerrar el defecto quirúrgico, no se crea una cavidad cerrada contaminada, sumado a que utilizamos antibiótico profilaxis para gram positivos y gram negativos en el intra y postoperatorio.

Adicionalmente, tuvimos un tiempo operatorio promedio de $39,2 \pm 20,5$ minutos. Por ser un procedimiento breve, utilizamos en la mayoría de los pacientes anestesia ra-

TABLA 4: TRABAJOS COMPARATIVOS

\begin{tabular}{|c|c|c|c|c|c|c|c|c|c|c|}
\hline \multirow[t]{2}{*}{ AUTORES } & \multirow{2}{*}{$\begin{array}{c}\text { PACIENTES } \\
\text { (n) }\end{array}$} & \multirow{2}{*}{$\begin{array}{l}\text { Morbilidad } \\
\text { Global (\%) }\end{array}$} & \multicolumn{2}{|c|}{ Morbilidad (\%) } & \multicolumn{2}{|c|}{ Sangrado (\%) } & \multicolumn{2}{|c|}{ Infección (\%) } & \multicolumn{2}{|c|}{ Re-operación (\%) } \\
\hline & & & $\mathrm{C}$ & $A$ & $\mathrm{C}$ & $A$ & C & $A$ & $\mathrm{C}$ & $A$ \\
\hline RAMIREZ & 40 & 10 & 15 & 5 & 0 & 0 & 5 & 5 & 0 & 0 \\
\hline HANLOSER & 75 & 19 & 12,5 & 17,1 & 3 & 11 & 10 & 6 & 2,5 & 0 \\
\hline NOURA & 43 & 18,6 & 33,3 & 1,5 & 23,8 & 0 & 4,6 & 0 & 19 & 0 \\
\hline BROWN & 341 & 11,7 & 8,4 & 19 & 4,7 & 7,6 & 2,1 & 6,7 & 0,4 & 1,9 \\
\hline LEE & 220 & 13,6 & 12 & 15 & 9 & 5 & 5 & 3 & 3 & 2 \\
\hline
\end{tabular}

C: defecto cerrado. A: defecto abierto 
quídea, lo cual nos permitió que reiniciaran rápidamente la ingesta oral y la deambulación, obteniendo un tiempo de internación promedio de $33 \pm 28,2$ horas.

Todos los pacientes recibieron controles endoscópicos por lo menos hasta el noveno mes de la cirugía y no hubo estenosis postoperatorias, incluso con resecciones de lesiones de $9 \mathrm{~cm}$ de diámetro. Es muy difícil cerrar este tipo de defectos sin estrechar la luz rectal, lo cual no sucede al dejar abierto el lecho y esperar el cierre por segunda intención.

A pesar de realizar todas las resecciones de espesor completo de la pared, no tuvimos perforaciones dentro de la cavidad abdominal, informadas en otras series en alrededor del $6 \%$ de los casos. ${ }^{40,41}$ Esto es debido, en parte, a que seleccionamos pacientes con lesiones de recto medio e inferior, haciendo que la posibilidad de esta complicación sea mucho menor. Si bien las tasas de mortalidad en este tipo de intervenciones son bajas, están reportadas en hasta un $2 \% \cdot{ }^{42,43}$ No tuvimos mortalidad en nuestra serie.

$\mathrm{Al}$ analizar nuestros resultados y teniendo en cuenta que en la mayoría de los trabajos no se observan diferencias significativas entre los que se cerró y se dejó abierta la brecha quirúrgica, cabría replantearse el beneficio de cerrar el defecto rectal, siendo esta una etapa laboriosa de la cirugía, que prolonga la misma y que presenta similares tasas de complicaciones. Adicionalmente es factible que, al ser técnicamente menos demandante, un mayor número de cirujanos puedan realizar este tipo de intervenciones. Se obtienen tiempos quirúrgicos más cortos, una pronta tolerancia a la ingesta oral y deambulación, posibilitando períodos de internación postoperatorios breves.

La limitación de este trabajo es que consistió en un estudio descriptivo, no pudiendo realizar comparación con los posibles resultados cerrando la brecha quirúrgica con un mismo equipamiento, técnica y equipo quirúrgico. De todas formas, los resultados de este estudio sirven como primer paso, planteando la necesidad de realizar un estudio comparativo entre las diferentes técnicas para llegar a conclusiones más claras en cuanto a sus beneficios.

\section{CONCLUSIÓN}

Los resultados de nuestro estudio permiten describir a la técnica de TAMIS dejando la brecha quirúrgica abierta en lesiones de recto extraperitoneal, como una técnica con buenos resultados en su implementación en nuestra población, con alta factibilidad y baja tasa de complicaciones, facilitando así la técnica quirúrgica.

\section{AGRADECIMIENTOS}

Quiero agradecer en primer lugar al Dr. Jorge Reales, quien fue mi maestro. Me introdujo en la coloproctología y me enseñó las técnicas y beneficios de la resección transanal. En segundo lugar, a mis compañeros del Centro de Estudios Digestivos, de la Clínica Pueyrredón y del Hospital Interzonal General de Agudos "Oscar Alende", por ayudarme en las cirugías y control de los pacientes, así como por impulsarme y colaborar en la elaboración de este trabajo.

\section{REFERENCIAS}

1. Lakkis Z, Manceau G, Bridoux V, Brouquet A, Kirzin S, Maggiori L, et al. French Research Group of Rectal Cancer Surgery (GRECCAR) and the French National Society of Coloproctology (SNFCP). Management of rectal cancer: the 2016 French guidelines. Colorectal Dis 2017 19:115-22.

2. Monson JR, Weiser MR, Buie WD, Chang GJ, Rafferty JF, Buie $\mathrm{WD}$, et al. Practice parameters for the management of rectal cancer (revised). Dis Colon Rectum. 2013;56(5):535-50.

3. Bryant CLC, Lunniss PJ, Knowles CH, Thaha MA, Chan CLH Anterior resection syndrome. Lancet Oncol 2012;13:403-08.

4. Abdelli A, Tillou X, Alves A, Menahem B. Genito-urinary sequelae after carcinological rectal resección: What to tell patients in 2017. J Visc Surg 2017; 154:93-104.

5. Morris E, Quirke P, Thomas JD, Fairley L, Cottier B, Foreman D. Unaceptable variation in abdominoperineal excision rates for rectal cancer: time to intervene? Gut 2014;57:1690-97.

6. Shingo N, Masayuki O, Norikatsu M, Masayoshi. Significance of defect closure following transanal local full-thickness excision of rectal malignant tumors. Mol Clin Oncol 2016;5:449-54.

7. Buess $G$, Theiss $R$, Günther $M$, Hutterer F, Pichlmaier $H$. Endoscopic surgery in the rectum. Endoscopy 1985;17:31-5.

8. Moore JS, Cataldo PA, Osler T, Hyman NH. Transanal endoscopic microsurgery is more effective than traditional transanal excision for resection of rectal masses. Dis Colon Rectum 2008;51:1026-31.

9. Casadeus D. Surgical resection of rectal adenoma: a rapid review. World J Gastroenterol 2009;15:3851-54.
10. Maya A, Vorenberg A, Oviedo M, da Silva G, Wexner SD, Sands D. Learning curve for transanal endoscopic microsurgery: a singlecenter experience. Surg Endosc 2014;28:1407-12.

11. Barendse RM, Dijkgraaf MG, Rolf UR, Bijnen AB, Consten EC, Hoff C, et al. Colorectal surgeons' learning curve of transanal endoscopic microsurgery. Surg Endosc. 2013; 27:3591-3602.

12. Atallah S, Albert M, Debeche-Adams T, Larach S. Transanal minimally invasive surgery (TAMIS): aplications beyond local excision. Tech Coloproct 2013;17:239-43.

13. Perez RO, Habr-Gama A, Lynn PB, São Julião GP, Bianchi R, Proscurshim I, et al. Transanal endoscopic microsurgery for residual rectal cancer (ypT02) following neoadjuvant chemoradiation therapy: another word of caution. Dis Colon Rectum 2013;56:6-13.

14. Sevá-Pereira G,Trombeta VL, Capochim Romagnolo LG. Transanal minimally invasive surgery (TAMIS) using a new disposable device: our initial experience. Tech Coloproctol 2014;18:393-97.

15. Menahem B, Alves A, Morello R, Lubrano J. Should the rectal defect be closed following transanal local excision of rectal tumors? A sistematic review and meta-analysis. Tech Coloproctol 2017;21:929-36.

16. Brown C, Raval MJ, Phang PT, Karimuccin A. The surgical defect after transanal endoscopic microsurgery: open versus closed managment. Surg Endosc 2016;31: 1078-82.

17. Brown C, Hochman D, Raval MJ, Moloo H, Phang PT, Bouchard A, et al. A multi-centre randomized controlled trial of open vs closed managment of the rectal defect after transanal endoscopic 
microsurgery. Colorectal Dis 2019;21:1025-31.

18. Wilhelm P, Storz P, Axt S, Falch C, Kirschniak A, Muller S. Use of self-retaining barbed suture for rectal wall closure in transanal endoscopic microsurgery. Tech Coloproctol 2014;18:813-16.

19. Lee L, Althoff A, Edwards K, Albert M, Atallah S, Hunter I, et al. Outcomes of closed versus open defects after local excision of rectal neoplasms: a multi-institutional matched analysis. Dis Colon Rectum 2018;61:172-78.

20. Hahnloser D, Cantero R, Salgado G, Dindo D, Rega D, Delrio P. Transanal minimal invasive surgery for rectal lesions: should the defect be closed. Colorectal Dis 2015;17:397-402.

21. Albert M, Atallah S, deBeche-Adams T, Izfar S, Larach S. Transanal minimally invasive surgery (TAMIS) for local excision of benign neoplasms and early-stage rectal cancer: efficacy and outcomes in the first 50 patients. Dis Colon Rectum 2013;56:301-07.

22. Marks J, Reynolds H. Local treatment of rectal cancer (TEM versus TAMIS versus transanal excision). In: Steele SR, Maykel JA, Champagne BJ, Orangio GR. (eds) Complexities in Colorectal Surgery: decision-making and management. Springer: New York. 2014. pp 219-230.

23. Atallah S, Albert M, Larach S. Transanal minimally invasive surgery: a giant leap forward. Surg Endosc 2010;24:2200-05.

24. Morino M, Allaix ME. Transanal endoscopic microsurgery: what indications in 2013? Gastroenterol Rep (Oxf) 2013;1:75-84.

25. Ramirez JM, Aguilella V, Gracia JA, Ortego Jr; Escudero P, Valencia $\mathrm{J}$, et al. Local fullthickness excision as first line treatment for sessile rectal adenomas: long- term results. Ann Surg 2009;249:225-28.

26. Ramirez JM, Aguilella V, Arribaas D, Martinez M. Transanal fullthickness excision of rectal tumors: should the defect be sutured? A randomized controlled trial. Colorectal Dis 2002;4:51-5.

27. Glimelius B, Tiret B, Cervantes A, Arnold D. Rectal cancer: ESMO Clinical Practice Guidelines for diagnosis, treatment and followup. Ann Oncol 2013 (Supp 6):S81-S8.

28. Burdan F, Sudol-Szopinska I, Staroslawska E, Kolodziejczak M, Klepacz $\mathrm{R}$, Mocarska A, et al. Magnetic resonance imaging and endorectal ultrasound for diagnosis of rectal lesion. Eur J Med Res 2015;20:1-4.

29. Balyasnikova S, Brown G. Optimal imaging strategies for rectal cancer staging and ongoing management. Curr Treat Options in Oncol 2016;17:32.

30. Garcia-Aguilar J, Mellgren A, Sirivongs P, Buie D, Madoff RD, Rothenberger DA. Local excision of rectal cancer without adjuvant therapy: a word of caution. Ann Surg 2000;231:345-51.

31. Perez M, Ribero A, Hunter L, Atallah S. A systematic review of transanal minimally invasive surgery (TAMIS) from 2010 to 2013.
Tech Coloproctol 2014;18:775-88.

32. Gill S, Stetler JL, Patel A, Shaffer VO, Srinivasan J, Staley C, et al. Transanal minimally invasive surgery (TAMIS): Standardizing a reproducible procedure.J Gastrointest Surg 2015;19:1528-36.

33. Lim SB, Seo SI, Lee JL, Kwak JY, Jang TY, Kim CW, et al. Feasibility of transanal minimally invasive surgery for mid-rectal lesions. Surg Endosc 2012;26:3127-32.

34. Lorenz C, Nimmesgern T, Back M, Langwieler TE. Transanal single port microsurgery (TSPM) as a modified technique of transanal endoscopic microsurgery (TEM). Surg Innov 2010;17:160-63.

35. Ragupathi M, Haas EM. Transanal endoscopic videoassisted excision: application of single-port access. JSLS 2011;15:53-8.

36. Noura S, Ohue M, Miyoshi N, Yasui M. Significance of defect closure following transanal local full-thickness excision of rectal malignant tumors. Mol Clin Oncol 2016;5: 449-54.

37. Bignell MB, Ramwell A, Evans JR, Dastur N, Simson JN. Complications of transanal endoscopic microsurgery (TEMS): a prospective audit. Colorectal Dis 2010;12:99-103.

38. Perez Oliva R, Habr-Gama A, Sao Juliao GP, Proscurshim I, Scanavini Neto A, Gama-Rodrigues J. Transanal endoscopic microsurgery for residual rectal cancer after neoadjuvant chemoradiation therapy is associated whit significant immediate pain and hospital readmission rates. Dis Colon Rectum 2011;54:545-51.

39. Issa N, Murninkas A, Schmilovitz-Weiss H, Agbarya A, Powsner E. Transanal endoscopic microsurgery after neoadjuvant chemoradiotherapy for rectal cancer. J Laparoendosc Adv Surg Tech 2015;25:617-24.

40. Rullier E, Rouanet P, Tuech JJ, Valverde A, Lelong B, Rivoire $\mathrm{M}$, et al. Organ preservation for rectal cancer (GRECCAR2): a prospective, randomised, open-label, multicentre, pase 3 trial. Lancet 2017;390: 469-79.

41. Morino M, Allaix ME, Famiglietti F, Caldart M, Arezzo A. Does peritoneal perforation affect short and long-term outcomes after transanal endoscopic microsurgery? Surg Endosc 2013;27:181-88.

42. Eyvazzadeh DJ, Lee JT, Madoff RD, Mellgren AF, Finne CO. Outcomes after transanal endoscopic microsurgery with intraperitoneal anastomosis. Dis Colon Rectum 2014;57:438-41.

43. Farmer KC, Wale R, Winnett J, Cunningham I, Grossberg P, Polglase A. Transanal endoscopic microsurgery: the first 50 cases. ANZ J Surg. 2002;72:854-56.

44. De Graaf EJ, Doornebosch PG, Stassen LP, Debets JM, Tetteroo GW, Hop WC. Transanal endoscopic microsurgery for rectal cancer. Eur J Cancer 2002;38:904-10.

\section{COMENTARIO}

Desde su aparición hace ya 10 años, la cirugía transanal mini-invasiva (TAMIS) descripta por Atallah y colaboradores determinó un cambio de paradigma en el manejo de los tumores de recto, siendo hoy en día un standard para ciertas patologías rectales, dejando en un segundo plano a la cirugía abdominal y a la cirugía transanal convencional. Numerosos trabajos describen los beneficios del TAMIS por sobre estas dos técnicas en casos bien seleccionados. Sin embargo, una menor cantidad de estudios analizan los resultados de este procedimiento con o sin cierre de la brecha quirúrgica.

Todo esto agrega aún más interés a la presente publicación del Dr. Diego Naiderman que con 35 casos operados sin cierre de la pared, obtuvo resultados en línea con las series publicadas. Cabe destacar la importancia de saber la ubicación exacta del defecto parietal, ya que si es sobre la cara anterior las chances de complicaciones postoperatorias son más probables.

Como muestra del interés actual que existe al respecto, las resecciones transanales fueron el tema elegido para el Relato Oficial del Congreso Argentino de Coloproctología 2019 desarrollado por el Dr. Gustavo Rossi en el que describe y analiza al TAMIS como una opción terapéutica con baja morbilidad y buenos resultados oncológicos. A su vez, a nivel nacional, los Dres. Minetti y col., el presente autor Naiderman y col., Amarillo y col., y Moreira Grecco y col. también publicaron sus experiencias en la resección transanal mini-invasiva. Entre estos autores, la decisión en cuanto al cierre de la brecha quirúrgica permanece dividida.

Sebastián Guckenheimer

Hospital Pirovano. C.A.B.A., Buenos Aires, Argentina. 